\title{
The analysis system COGITAT for the study of cognitive deficiencies in rodents
}

\author{
CHRISTINE HEIM \\ Max Planck Institute for Experimental Medicine, Göttingen, Germany \\ IANCU PARDOWITZ \\ Fa. Cognitron GmbH, Göttingen, Germany \\ MARIA SIEKLUCKA \\ Lublin Medical University, Lublin, Poland \\ WACLAV KOLASIEWICZ \\ Institute of Pharmacology, Polish Academy of Sciences, Krakow, Poland \\ THOMAS SONTAG \\ Veterinary-Biological Laboratory, Veterinary Clinic, Northeim, Germany \\ and \\ KARL-HEINZ SONTAG \\ Max Planck Institute for Experimental Medicine, Göttingen, Germany
}

\begin{abstract}
COGITAT is an automated hole board system that, following minimal experimental interventions, makes it possible to measure a variety of parameters associated with learning, memory, relearning, cognition, and cognitive shifts, but also changes in exploratory and sensorimotor performance in rodent models. The individual parameters-that is, overall exploratory activity, number of visits (deep in the hole) into or inspections of (at the upper surface) holes, number of baited, unbaited, or previously baited holes visited or inspected, reinspections of or revisits into any holes, number of pellets eaten, time to find pellets, serial order collection (without intermediate inspections or visits), and reference and working memory errors (visits, inspections, or total)-are obtained simultaneously, and the results are immediately available after the end of each experiment. The system appears to be well suited to neurophysiological, neuropharmacological, and gene-technological investigations in rodent models.
\end{abstract}

The rapid growth of molecular genetic studies of the nervous system offers an exciting opportunity to begin to understand the complex consequences of genetic perturbations on cognitive, emotional, rewarding, or degenerative behavior patterns and related disease states, such as movement disorders, inherited degenerative disorders, or Alzheimer's disease. Research has to focus on the functional outcomes of such perturbations, and such studies may lead to explanations of how behavior is controlled at both the behavioral and the neurophysiological levels.

It is always a problem to analyze disturbances of a physiologically functioning system in such a way that one may

This study was supported by the Bundesministerium für Bildung, Wissenschaft, Forschung und Technologie, BMBF-Schwerpunkt "Morbus Parkinson und andere Basalganglienerkrankungen," Grant 01 KL 9403/0. The skillful technical assistance of Heike Wetzstein is gratefully acknowledged. We thank two anonymous reviewers for their helpful criticism of a preliminary version of this manuscript. Correspondence concerning this article should be addressed to C. Heim, Department of Neuropharmacology and -physiology, Max Planck Institute for Experimental Medicine, Hermann-Rein-Str. 3, D-37075 Göttingen, Germany (e-mail: sontag@exmda2.mpiem.gwdg.de). make valid comparisons between its pathological and its normal activity. Initially, the question arises of how one can test the animals' capabilities when faced with a new environment and new tasks. Experimental setups need to be designed to be within the normal range of natural behavior. The experimenter has to be sure that the animals are given the opportunity to find the answers to the problems with which the test procedure confronts them.

All such studies are time consuming, and the results are often inconsistent from one experiment to another (Eijkenboom \& van der Staay, 1998) and from one laboratory to another (Bear, 1999). Moreover, up till now, different testing procedures have had to be used to measure cognitive and motor capabilities, working and reference memory, serial learning, and shifting aptitude. Since the different test procedures have had to be carried out one after the other, it has not hitherto been possible to obtain a simultaneous and comprehensive impression of which parameters might be affected by some particular intervention. Furthermore, sequential studies are bound to involve different circumstances and environmental conditions, which will affect the animals in different ways and, again, 
prevent the experimenter from obtaining and correctly interpreting a strictly comparable and complete view of how the animals are coping with their deficits at any instant in time following some previous intervention (see also Spruijt, Buma, van Lochem, \& Rouseau, 1998).

In an attempt to get much closer to a solution of these problems, we have elaborated an enlarged version of a hole board (Oades \& Isaacson, 1978). This system permits the detailed and automatic simultaneous recording of cognitive and motor behavior-- that is, cognition and recognition, reference learning, memory deficits, working and reference memory, and the application and variation of successive search strategies and their sequence, shifting disabilities, and/or loss of motivation or attention, and sensorimotor defects in animal models.

Oligemic episodes and increased iron concentrations in discrete brain regions have been reported to occur with advancing age and are thought to be associated with neurodegenerative diseases (Connor, 1992; Heim, Zhang, et al., 2000; Nakano, Kogure, \& Fujikura, 1990). In order to follow the development of event-related deficiencies, whose appearance may be delayed, progressive, or both, and in order to gain an accurate impression of the time course of such abnormal behavior patterns as may arise, it is necessary, at various times, to follow many different behavioral parameters simultaneously under comparable conditions.

In order to demonstrate the differential effects of different experimental interventions on some measured parameters, an abridged description of the experimental system follows, which presents the manifold possible ways of recording offered by the equipment, shows all the measurement parameters and recording modes, and appends a small selection of particularly noteworthy parameters, either after a single cerebral oligemic episode or also after a subsequent intrastriatal or intranigral injection of iron.

\section{The COGITAT Hole Board}

During a test period, rats explore a COGITAT hole board (size, $825 \times 825 \mathrm{~mm}$, inner surface; height, $350 \mathrm{~mm}$ ) bordered by a clear Plexiglas surround (height, $270 \mathrm{~mm}$ ) giving access to distal spatial cues. The board contains 25 holes (diameter, $60 \mathrm{~mm}$; distance apart center to center, $165 \mathrm{~mm}$ ), each consisting of a cylindrical tube that is closed off at its lower end by an adjustable feeding plate $(60,75$, or $90 \mathrm{~mm}$ below the upper surface, depending on the size of the animals) with a depression into which a food pellet $(0.045 \mathrm{~g}$; Bio-Serv, Frenchtown, $\mathrm{NJ})$ fits exactly. The color of the feeding plate and of the food pellet is a perfect match. The ground below the feeding plate and the cylindrical tubes is covered with the same pellets, to prevent the animals from working out the pattern of the distribution of the pellets by using olfactory stimuli. In a typical experimental run, for example, eight of the cylinders are baited with pellets, one for each hole. A run (trial) is automatically ended after a predefined time period. During the time-limited phase, the animals are provided with the opportunity of finding and eating the food pellets. At the same time, however, they also have the opportunity of recognizing the spatial pattern in which the pellets are presented and of remembering this pattern.

The system uses an infrared system to record activity: differential dips of the head at the upper level of the tubes $(10 \mathrm{~mm}$ beneath the upper surface), which are equivalent to inspections; exploration deep into the hole ( $20 \mathrm{~mm}$ above the level of the pellet), but without eating a pellet, which are scored as visits; and collections of the pellets by eating them, which is detected by the interruption of a beam of infrared light at the level of the pellet.

Table 1 shows the manifold, partly dependent parameters, which can be automatically and simultaneously recorded.

Supplementary to these predefined and automatically presented parameters, the experimenter can obtain information about the average percentage of a group's visits into or inspections of holes that are right at the edge of the board, enabling him to differentiate between searching strategies that are mainly tactile cue directed (via thigmotaxis) and those that are more visual cue directed and/or egocentric. Similarly, one can ascertain whetherfor example, following an experimental local intracerebral lesion - the attention of the animals during the search is directed more to the ipsilateral or the contralateral side, which enables one to ascertain lesion-originating sensorimotor deficits. All this is made possible by defining the holes of interest as an additional pattern, and putting them in relation to the overall exploratory activity. Additional surveillance with a video camera may offer the possibility of recording not only the correct path and speed of the animals, but also their body and head posture and their motor performance.

Daily random switching from different distinct patterns-for example, from A to B (see Figure 5C) by turns - is another analytical procedure; which enables the experimenter not only to calculate how many hits or misses the animal requires to recognize which actual pattern it has to use on any given day, but also to determine whether success has been achieved by getting it right or merely by chance. For orientation, the first three holes of a known pattern of holes baited or unbaited may already, depending on the animals' cognitive capabilities, provide them with enough information to recognize which pattern is being presented.

COGITAT is also able to help to differentiate between failure of perception and failure of memory or performance. This can be done by using the time-to-learn-fit (see Figure 9), where the steepness of the sigmoid curve can give information about the capability of the animals to recognize the task (perception).

The software of COGITAT (COGITAT R.N.5, Cognitron $\mathrm{GmbH}$, Göttingen, Germany) runs on Windows 95 
Table 1

List of Parameters That Can Be Obtained Automatically by the COGITAT System

\begin{tabular}{|c|c|}
\hline Parameter & Definition/Explanation \\
\hline Overall exploratory activity & The sum of inspected and visited holes, with and without food collection \\
\hline Inspections & The number of holes inspected by interruption of the upper beam \\
\hline Visits & The number of holes visited by interruption of the upper and lower beam \\
\hline Inspections of baited holes & The number of baited holes inspected by interruption of the upper beam \\
\hline Inspections of unbaited holes & The number of unbaited holes inspected by interruption of the upper beam \\
\hline Visits to baited holes & The number of visits to baited holes by interruption of the upper and lower beam \\
\hline Visits to unbaited holes & The number of visits to unbaited holes by interruption of the upper and lower beam \\
\hline Pellets eaten & The number of pellets eaten \\
\hline Reinspections of previously baited holes & The number of reinspections of previously baited holes \\
\hline Revisits of previously baited holes & The number of times previously baited holes were revisited \\
\hline Reinspections of any holes & The number of reinspections of any holes (unbaited and/or baited) \\
\hline Revisits of any holes & The number of times any holes (unbaited and/or baited) were revisited \\
\hline Working memory errors, total & $\begin{array}{l}\text { The percentage of the sum of inspections and visits to previously baited holes in relation to the total } \\
\text { number of holes inspected and visited }\end{array}$ \\
\hline Reference memory errors, total & $\begin{array}{l}\text { The percentage of the sum of inspections and visits to unbaited holes in relation to the total number } \\
\text { of holes inspected and visited }\end{array}$ \\
\hline $\begin{array}{l}\text { Working memory errors, } \\
\text { perfunctory inspections }\end{array}$ & $\begin{array}{l}\text { The percentage of inspections of previously baited holes in relation to the total number of holes } \\
\text { inspected }\end{array}$ \\
\hline $\begin{array}{l}\text { Reference memory errors, } \\
\text { perfunctory inspections }\end{array}$ & The percentage of inspections of unbaited holes in relation to the total number of holes inspected \\
\hline Working memory errors, visits & The percentage of visits to previously baited in relation to the total number of holes visited \\
\hline Reference memory errors, visits & The percentage of visits to unbaited holes in relation to the total number of holes visited \\
\hline Serial collection & $\begin{array}{l}\text { The number of holes that were baited with pellets that were emptied in a serial order without inter- } \\
\text { mediate inspections or visits in other holes than those in the pattern }\end{array}$ \\
\hline Time to find pellets & The time it took to find the pellets within a time limit \\
\hline Display of food collection & $\begin{array}{l}\text { The path taken by each animal while performing the task and a display of inspections or visits to } \\
\text { baited and unbaited holes, indicating the actual pattern and the collection of the pellets }\end{array}$ \\
\hline Time to learn & $\begin{array}{l}\text { The number of trials until optimal performance-that is, finding all pellets (parameter for recognition } \\
\text { of the task) }\end{array}$ \\
\hline Shifting aptitude & $\begin{array}{l}\text { Immediately after having acquired Pattern A, the animals are confronted with a new pattern and have } \\
\text { to unlearn the originally learned pattern and learn the new one. What is recorded is the number of } \\
\text { holes learned that are appropriate to the new pattern and the number that is still related to the old } \\
\text { one, from which data learning and forgetting curves can be constructed. }\end{array}$ \\
\hline
\end{tabular}

Note-All parameters are simultaneously available immediately after the end of the experiment(s). Parameters 1-20 are listed as results files for immediate calculation by using, for example, SAS.

or Windows NT. The operator works with a user-friendly graphical interface to analyze and display information about all the parameters recorded by the system.

\section{METHOD}

\section{Animals}

For surgery, male 3-4-month-old Wistar rats weighing 330-400 g were used. The animals were kept on a 12:12-h light:dark cycle, and humidity and room temperature were $55 \%$ and $21^{\circ} \mathrm{C}$, respectively. Food and water were provided ad lib.

\section{Surgery}

Results are shown for the following four groups. Groups 1: Nine rats were subjected to a transient reduction in cerebral blood flow to oligemic levels by bilateral clamping of their carotid arteries (BCCA) for $60 \mathrm{~min}$ under pentobarbital anesthesia $(60 \mathrm{mg} / \mathrm{kg}$; Nembutal, Sanofi, France). Rectal temperature was approximately $37^{\circ} \mathrm{C}$, and the mean arterial blood pressure always showed normotensive values during surgery. After an occlusion time of $60 \mathrm{~min}$, the clamping threads were removed, restoration of blood flow was visually inspected, and the surrounding skin was sutured (for further details, see Block, Sieklucka, Schmidt-Kastner, Heim, \& Sontag, 1993; Block, Szabo, Jaspers, Heim, \& Sontag, 1993). The reduction of cerebral blood flow induced no cerebral necrotic processes (Heim \& Sontag, 1994; Melzacka et al., 1994), such as those found in models used for studying cerebral stroke processes after an additional decrease in blood flow to ischemic levels (M. L. Smith, Auer, $\&$ Siesjö, 1984). Group 2: Control rats $(n=8)$ were sham operated by having their carotid arteries prepared but not clamped.

Additional groups of animals were administered either doses of $35 \mathrm{ng} \mathrm{FeCl}$ dissolved in $0.25 \mu$ iso-osmotic citrate buffer (Sengstock, Olanow, Dunn, \& Arendash, 1992) bilaterally into the substantia nigra (bcfeSN; Group 3; $n=12$ ), using coordinates (according to Paxinos \& Watson, 1982) bregma $-5.3 \mathrm{~mm}, 2.3 \mathrm{~mm}$ laterally and $7.5 \mathrm{~mm}$ below the cortical surface, $1.5 \mu \mathrm{g} \mathrm{FeCl}_{3}$ in $2 \mu \mathrm{l}$ buffer unilaterally into the ventrolateral striatum (bcfestria; Group $4 ; n=$ 12), using coordinates $0.2 \mathrm{~mm}$ anterior to bregma, $4.0 \mathrm{~mm}$ laterally and $7.0 \mathrm{~mm}$ below the cortical surface, 1 week following BCCA. For a direct comparison within the predestined experimental groups, BCCA-treated and sham-operated rats also received a bilateral injection of buffer 1 week after the BCCA or the sham operation.

The four subgroups of animals presented here were taken from two experimental groups of rats out of a total of $n=82$, where the animals were given small iron injections under pentobarbital anesthesia. The iron was injected either bilaterally into the substantia nigra or unilaterally into the ventrolateral striatum, with (as was mentioned above) or without a previous transient oligemic episode to the forebrain induced by BCCA 1 week beforehand, to mimic basal ganglia disorders (Arendash, Olanow, \& Sengstock, 1993; Heim, Kolasiewicz, \& Sontag, 2000; Kolasiewicz et al., 1995). Corresponding control rats were injected with the same amount of iron vehicle (but devoid of iron) either intrastriatally or intranigrally 
I week after BCCA or after a sham operation. Each experimental group consisted of $n=41$ rats. We present two particularly interesting examples for demonstration purposes, in order to illustrate the capabilities of the system. It would take too long to present the complete analysis of the actual behavioral profiles recorded for all the subgroups of the complete animal collective after a variety of different interventions over a period of 21 months. The results of investigations obtained with the system in various specific research areas are being prepared for further publication.

\section{Feeding Procedure}

Animals intended for testing on the hole board were put on starvation rations a week beforehand and throughout the subsequent test periods. To reduce the stress caused by food restriction (see also Bear, 1999; Deroche et al., 1995) and subsequent changes in the balance of dopaminergic systems (Pothos, Creese, \& Hoebel, 1995 ), the reduction in food intake offered to the animals caused a weight reduction of not more than $\sim 10-15 \%$. The animals were fed $1 \mathrm{~h}$ before the start of the test procedure for the following reasons. When feeding was offered after the test procedure, animals awaiting their daily food ration after the hole board procedure did not search intensively enough during the trials. Moreover, feeding shortly before the test procedure removes the decrease in dopamine release within the nucleus accumbens that accompanies chronic food restriction (Pothos et al., 1995) - an aspect that seems most important when animal models with local disturbances of their dopaminergic systems are being tested.

\section{Test Procedure}

Spatial learning and memory were tested, beginning 3 months after surgery. The experiments were carried out between 7 and 10 a.m. Animals remaining in their home cages were adapted to the laboratory for $1 \mathrm{~h}$ and fed with approximately $12-15 \mathrm{~g}$ of laboratory chow. Eight holes of the hole board, assigned here, for example, to either Pattern A or Pattern B (Figure 5C), were serially baited with food pellets not visible to the animals while walking. The animals were then tested in a random manner. At the start of the experiment, the animals were allowed to adapt for $10 \mathrm{sec}$ in the starting box at the entrance of the board. A trial was completed as soon as the animal had collected and eaten all of the pellets within the allotted time span or when the allotted time span had elapsed, whichever was sooner. Animals with a good knowledge of the pattern are able to collect all eight pellets within an average time of $20 \mathrm{sec}$ (see also Heim \& Sontag, 1994).

Spatial cues available for the animals inside the hole board enclosure were the entrance with the starting box, the four corners of the enclosure, the upper edges of the 25 holes, and as tactile stimuli, the side walls, for orientation with the vibrissae (thigmotaxis). Outside the enclosure, distal cues visible through the transparent Plexiglas walls were, besides the experimenter, a computer, a laboratory clock, a window, two doors, two cupboards, two lines of tables with the cages of the remaining experimental animals on them, two lights, and, in the center above the hole board, the video camera attached to a beam at right angles.

At first, Pattern A (Figure 5C, black quadrants) was presented. Each animal was subjected to only a single trial per day. During the first experimental session, a trial duration of $180 \mathrm{sec}$ provided the animals with the opportunity to explore the hole board and to find eight hidden pellets. As soon as every animal of the corresponding control group had found all of the pellets at least twice, the time allowed was reduced to $60 \mathrm{sec}$ and, again, to $30 \mathrm{sec}$ per trial. The learning process was regarded as being complete when all of the control animals had succeeded in collecting all eight pellets during the allotted test period of $30 \mathrm{sec}$ at least twice .

This procedure was chosen for the following reasons. First, it was not initially known whether an experimental group would be capable of finding all of the pellets, and therefore, the success of the control group was regarded as being likely to be the optimal and, generally, the most convenient yardstick. Second, as the effects of intertrial intervals would possibly be an important factor for the achievements of the individual animals, we did not set the criterion for a rat to move to a shorter allotted time as the performance of any individual rat but, rather, to the performance of all the rats (of the control group).

Immediately after having acquired Pattern A (Figure 5C, black quadrants), the rats learned Pattern B (Figure 5C, gray quadrants), which is adjacent to two walls of the board enclosure, over 11 sessions of $30 \mathrm{sec}$. This test should provide answers about the flexibility of the tested rats, whether and when they are able to switch their attention from one location where food is presented to a second one.

\section{Statistical Procedure}

For statistical evaluation a two-factorial analysis of variance (ANOVA), with group and trial as factors, was used. The results are displayed as means $\pm S E M$ s of the individual daily trials of the corresponding experimental periods.

\section{RESULTS AND CONCLUSIONS}

During the animals' acquisition of the learning task, COGITAT provided the experimenter with at least 25 different parameters to study, and these supplied detailed information about differences in the animals' overall exploratory behavior and in their learning and memory capabilities, in a comparison between the BCCA-treated rats and the sham-operated controls, on the one hand, and the animals treated with iron - either intrastriatally or intranigrally-on the other.

The parameters that are measurable automatically are shown in Table 2, which depicts in which of the parameters BCCA-treated rats differed from sham-operated controls when they were confronted with the challenge of exploring a hole board, to find the food pellets hidden in it - at first by chance (incentive learning). Figures 1-4 show that, during the runs of $180 \mathrm{sec} /$ trial, $60 \mathrm{sec} / \mathrm{trial}$, and $30 \mathrm{sec} /$ trial, both the BCCA-treated and the shamoperated control animals were generally able to manage the task. A training or learning effect could be seen during the time course from 180- to 60- and 30-sec training sessions (Figures $1 \mathrm{~A}-1 \mathrm{C}$ and $1 \mathrm{D}-1 \mathrm{~F}$ ). However, during the first two training sessions of 180 - and $60-$ sec duration per trial, BCCA-treated rats found fewer pellets distributed according to Pattern $A$ in the given time [180 sec/ trial: group effect, $F(1,15)=6.10, p=.0148$; trial effect, $F(8,120)=5.61, p=.0001$; no group $\times$ trial interaction; $60 \mathrm{sec} /$ trial: group effect, $F(1,15)=4.92, p=$ .0287 ; no trial effect; no group $\times$ trial interaction; Figures $1 \mathrm{D}$ and $1 \mathrm{E}]$.

Figure 2 shows in panels $\mathrm{A}-\mathrm{C}$ that the overall exploratory activity exhibited increased values for the BCCA group, as compared with controls, after the time available was reduced to the next time limit $[180 \mathrm{sec} /$ trial: $n . s$.; $60 \mathrm{sec} /$ trial: group effect, $F(1,15)=5.11, p=.0259 ;$ no trial effect; no group $\times$ trial interaction; $30 \mathrm{sec} /$ trial: group effect, $F(1,15)=47.25, p=.0001$; no trial effect; no group $\times$ trial effect]. This increased exploratory activity provided a sufficient number of visits (Figures 
Table 2

Group Effects

\begin{tabular}{|c|c|c|c|c|}
\hline \multicolumn{2}{|r|}{ Parameter } & \multirow{2}{*}{$\frac{180 \mathrm{sec}}{\mathrm{B}>\mathrm{S}^{*}}$} & \multirow{2}{*}{$\frac{60 \mathrm{sec}}{\mathrm{n} . \mathrm{s} .}$} & \multirow{2}{*}{$\frac{30 \mathrm{sec}}{\text { n.s. }}$} \\
\hline 1 & Time to find pellets & & & \\
\hline 2 & Overall exploratory activity (OEA) & n.s. & $\mathrm{B}>\mathrm{S}^{*}$ & $\mathrm{~B}>\mathrm{S}_{+}^{+}$ \\
\hline 3 & Visits & n.s. & n.s. & n.s. \\
\hline 4 & Visits of baited holes & n.s. & n.s. & n.s. \\
\hline 5 & Pellets eaten & $\mathrm{B}<\mathrm{S}^{*}$ & $\mathrm{~B}<\mathrm{S}^{*}$ & n.s. \\
\hline 6 & Visits of unbaited holes & n.s. & $\mathrm{B}>\mathrm{S}^{*}$ & n.s. \\
\hline 7 & Revisits of previously baited holes & n.s. & n.s. & $\mathrm{B}>\mathrm{S}^{*}$ \\
\hline 8 & Repeated revisits of any holes & n.s. & n.s. & $\mathrm{B}>\mathrm{S}^{*}$ \\
\hline 9 & Serial collection & $\mathrm{B}<\mathrm{S} \dagger$ & $\mathrm{B}<\mathrm{S} \ddagger$ & $\mathrm{B}<\mathrm{S} \dagger$ \\
\hline 10 & Inspections & n.s. & $\mathrm{B}>\mathrm{S} \dagger$ & $\mathrm{B}>\mathrm{S}+$ \\
\hline 11 & Inspections of baited holes & n.s. & n.s. & $\mathrm{B}<\mathrm{S} \ddagger$ \\
\hline 12 & Inspections of unbaited holes & n.s. & $\mathrm{B}>\mathrm{S} \uparrow$ & $\mathrm{B}>\mathrm{S}+$ \\
\hline 13 & Reinspections of previously baited holes & n.s. & n.s. & $\mathrm{B}>\mathrm{S} \ddagger$ \\
\hline 14 & Repeated inspections of any holes & $\mathrm{B}>\mathrm{S}^{*}$ & $\mathrm{~B}>\mathrm{S}^{*}$ & $B>S \ddagger$ \\
\hline 15 & Working memory errors (WME) visits & n.s. & n.s. & $\mathrm{B}>\mathrm{S}^{*}$ \\
\hline 16 & Reference memory errors (RME) visits & n.s. & $\mathrm{B}>\mathrm{S}^{*}$ & n.s. \\
\hline 17 & Working memory errors \% of inspections & n.s. & n.s. & n.s. \\
\hline 18 & Reference memory errors \% of inspections & n.s. & n.s. & $\mathrm{B}>\mathrm{S}^{*}$ \\
\hline 19 & Working memory errors total & n.s. & n.s. & $\mathrm{B}>\mathrm{S} \dagger$ \\
\hline 20 & Reference memory errors total & $\mathrm{B}>\mathrm{S} \ddagger$ & $\mathrm{B}>\mathrm{S} \ddagger$ & $\mathrm{B}>\mathrm{S} \neq$ \\
\hline 21 & WME inspections \% of OEA & n.s. & n.s. & $\mathrm{B}>\mathrm{S} \dagger$ \\
\hline 22 & RME inspections \% of OEA & $\mathrm{B}>\mathrm{S}^{*}$ & $\mathrm{~B}>\mathrm{S} \dagger$ & $\mathrm{B}>\mathrm{S}+$ \\
\hline
\end{tabular}

Note-The table shows at a glance how BCCA-treated animals are distinguished from sham-operated animals in the three consecutive test periods of $180 \mathrm{sec}$ per trial, $60 \mathrm{sec}$ per trial, and $30 \mathrm{sec}$ per trial. Group differences over the whole range of test periods are presented. $180 \mathrm{sec}$ : period with 9 trials at $180 \mathrm{sec}$ per trial, 1 per day; $60 \mathrm{sec}$ : period with 7 trials of $60 \mathrm{sec}, 1$ per day; $30 \mathrm{sec}$ : period with 13 trials of $30 \mathrm{sec}, 1$ per day; B, BCCA; S, sham. Parameters $1-20$ are explained in Table 1 and are listed as results files for immediate calculation by using, for example, SAS. Parameters 21 and 22 have been calculated by dividing the number of reinspections of previously baited holes (WME) or of inspections of unbaited holes (RME) by the overall exploratory activity. ${ }^{*} p<.05 . \quad{ }^{\dagger} p<.01 . \quad{ }^{\ddagger} p<.001$.

2D-2F), which is not different from control values. The number of unrewarding inspections, however, showed increased values, as compared with controls [Figures $2 \mathrm{G}-2 \mathrm{I}$; $180 \mathrm{sec} /$ trial; n.s.; $60 \mathrm{sec} /$ trial: group effect, $F(1,15)=$ $19.82, p=.0028$; no trial effect; no group $\times$ trial interaction; $30 \mathrm{sec} /$ trial: group effect, $F(1,15)=51.44, p=$ .0001 ; no trial effect; no group $\times$ trial interaction].

A learning capability in the avoidance of unbaited holes or of revisiting previously baited holes can be seen for sham-operated as well as for BCCA-treated rats (Figures $3 \mathrm{~A}-3 \mathrm{C}$ and $3 \mathrm{D}-3 \mathrm{~F}$ ). This cognitive ability differed between the BCCA and the sham-operated animals with respect to time available: Shortening the time available from 180 to $60 \mathrm{sec}$ once again induced a transient increase in the number of visits to unbaited holes on the part of only the $\mathrm{BCCA}$-treated rats [Figure 3B; group effect, $F(1,15)=$ $3.99, p=.0485$; no trial effect; no group $\times$ trial interaction]. Revisits of previously baited holes showed significant differences, with increased values for the BCCA animals during the last $30 \mathrm{sec} /$ trial session [Figure $3 \mathrm{~F}$; group effect, $F(1,15)=6.46, p=.0118$; no trial effect; no group $\times$ trial interaction]. The shape of the slope exactly followed the time curve of revisits of any holes [not shown; group effect, $F(1,15)=6.16, p=.0139$; no trial effect; no group $\times$ trial interaction]. However, the mean values were below 1 , so it is questionable whether the revisits can be regarded as relevant at all.
Revisits or reinspections cannot be regarded as being part of some stereotyped behavior, since repeated head dips were not counted if an animal repeatedly moved its head several times in the same hole, and a second count was only counted if the animal had visited or inspected other holes in between.

At the end of the $30 \mathrm{sec} /$ trial training sessions, all of the rats were able to search successfully (Figures $1 \mathrm{C}$ and $1 \mathrm{~F}$ and $3 \mathrm{C}$ and $3 \mathrm{~F}$ ), but the $\mathrm{BCCA}$ rats showed a smaller decrease in their inspection of unbaited holes from one session to the next, as compared with the sham-operated rats [Figures $4 \mathrm{~A}-4 \mathrm{C}: 180 \mathrm{sec} /$ trial: n.s.; $60 \mathrm{sec} /$ trial: group effect, $F(1,15)=8.80, p=.0037$; no trial effect; no group $\times$ trial interaction; $30 \mathrm{sec} /$ trial: group effect, $F(1,15)=50.21, p=.0001$; no trial effect; no group $\times$ trial interaction], and this resulted in their collecting fewer pellets in a serial order up to the final trial [Figures $4 \mathrm{D}-4 \mathrm{~F}$; $180 \mathrm{sec} /$ trial: group effect, $F(1,15)=8.19, p=.0049$; trial effect, $F(8,120)=4.63, p=.0001$; no group $\times$ trial interaction; $60 \mathrm{sec} /$ trial: group effect, $F(1,15)=$ $19.82, p=.0001$; no trial effect; no group $\times$ trial interaction; 30 sec/trial: group effect, $F(1,15)=28.62, p=$ .0001 ; no trial effect; no group $\times$ trial interaction].

Reference memory errors visits were only significant during the $60 \mathrm{sec} /$ trial session [not shown; group effect, $F(1,15)=4.84, p=.0302$; no trial effect; no group $\times$ trial interaction]. The measurement of total reference mem- 

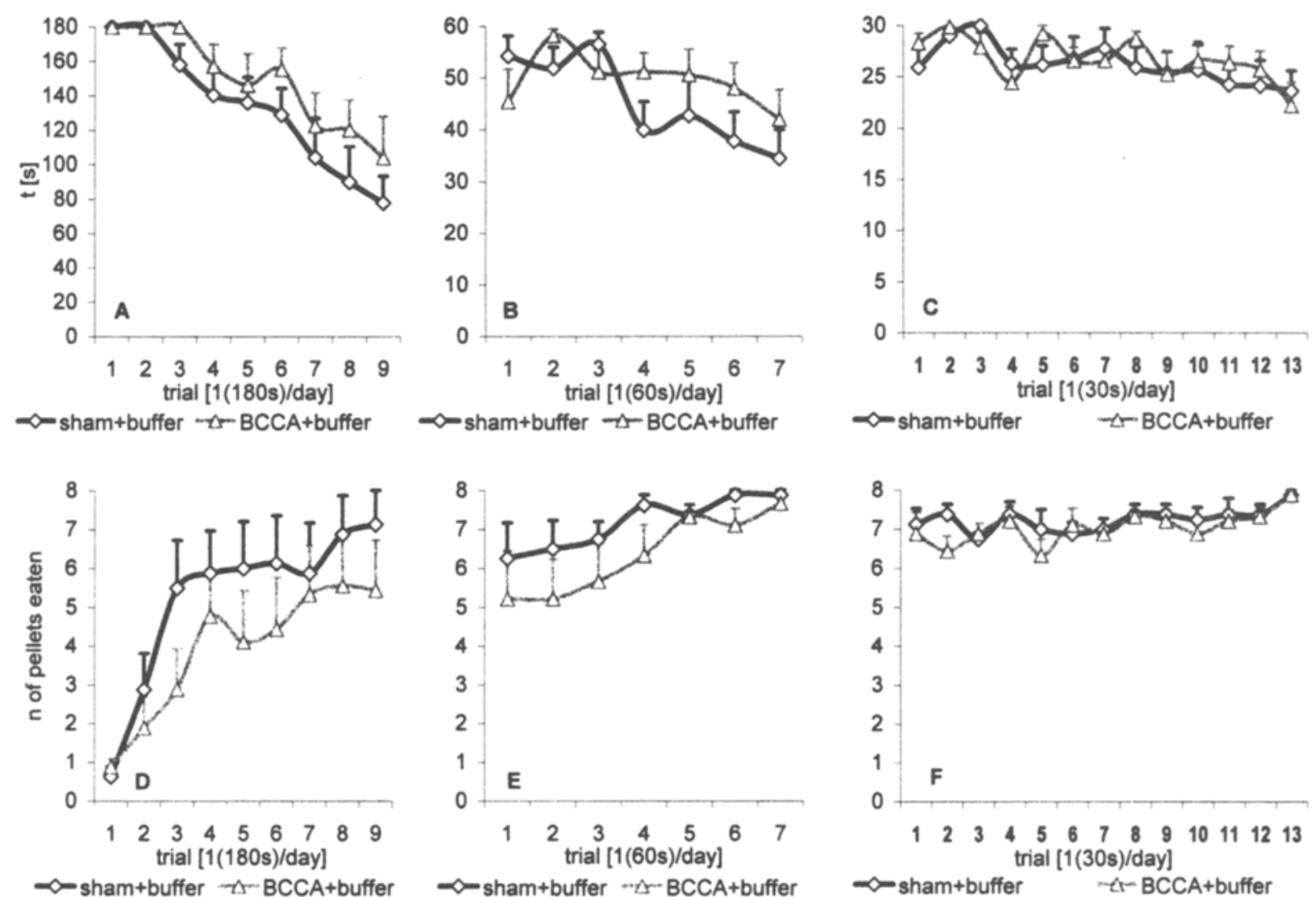

Figure 1. Curves illustrating the time taken to find the hidden pellets in the holes of Pattern A. Panels A and D: time required to find and eat a distinct number of pellets within $180 \mathrm{sec}$ per trial and per day. BCCA-affected rats found fewer pellets and needed more time for success, as compared with controls. Panels B and E: Following a reduction in the available time to 60 sec per trial per day, the BCCA rats still found fewer pellets over the whole time period $(E)$, whereas the time to find the pellets was no different. However, on Day 7, the BCCA rats had improved their success at finding pellets over Day 9 of the 180-sec trial. Panels $C$ and F: BCCA-affected rats had achieved the same success in finding and eating seven or eight pellets $(C)$ in the same time as the controls. BCCA-affected rats need more time for a successful performance of the task. Values are shown as mean $\pm S E M$; sham + buffer, $n=8$ rats; BCCA + buffer, $n=9$ rats.

ory errors and of reference memory errors involving inspections, both of which were calculated in relation to the overall exploratory activity, showed significant differences during all three trial sessions [not shown; reference memory errors, total $180 \mathrm{sec} /$ trial: group effect, $F(1,15)$ $=12.31, p=.0006$; trial effect, $F(8,120)=5.66, p=$ .0001 ; no group $\times$ trial interaction; $60 \mathrm{sec} /$ trial: group effect, $F(1,15)=16.79, p=.0001 ;$ no trial effect; no group $\times$ trial interaction; $30 \mathrm{sec} /$ trial: group effect, $F(1,15)=38.42, p=.0001$; no trial effect; no group $X$ trial interaction; reference memory errors, inspections in relation to the overall exploratory activity, $180 \mathrm{sec} /$ trial group effect, $F(1,15)=6.19, p=.0141$; trial effect, $F(8,120)=3.17, p=.0025$; no group $\times$ trial interaction; $60 \mathrm{sec} /$ trial: group effect, $F(1,15)=8.44, p=.0045$; no trial effect; no group $\times$ trial interaction; $30 \mathrm{sec} /$ trial: group effect, $F(1,15)=42.11, p=.0001$; no trial effect; no group $\times$ trial interaction].

If one takes Figures $1-4$ together and also includes the results of the reference memory errors, it appears that the
BCCA animals already have a deficit in their reference memory during the $180 \mathrm{sec} /$ trial period, for which they are, however, able to compensate by an increased locomotor and exploratory activity during the following sessions with a reduced available time. This compensation apparently resulted in the same learning success-measured as the number of pellets consumed-as that of the sham-operated animals. The fact that the animals had recognized the task could be ascertained with reference to the time-to-learn curve, which showed the same steepness of rise as that of the sham-operated animals (not shown).

The increased exploratory activity cannot be regarded as a general defect in locomotor activity, since it is not obvious during the very first trial session of $180 \mathrm{sec} /$ trial (Figure 2A). Comparing the slopes for overall exploratory activity (Figures $2 \mathrm{~A}-2 \mathrm{C}$ ) and inspections (Figures 2G-2I) with the slopes for time to find pellets (Figures $1 \mathrm{~A}-1 \mathrm{C}$ ) and the number of pellets eaten (Figures $1 \mathrm{D}-1 \mathrm{~F}$ ), it appears that the BCCA animals increased their explorations only after they had recognized that a distinct num- 

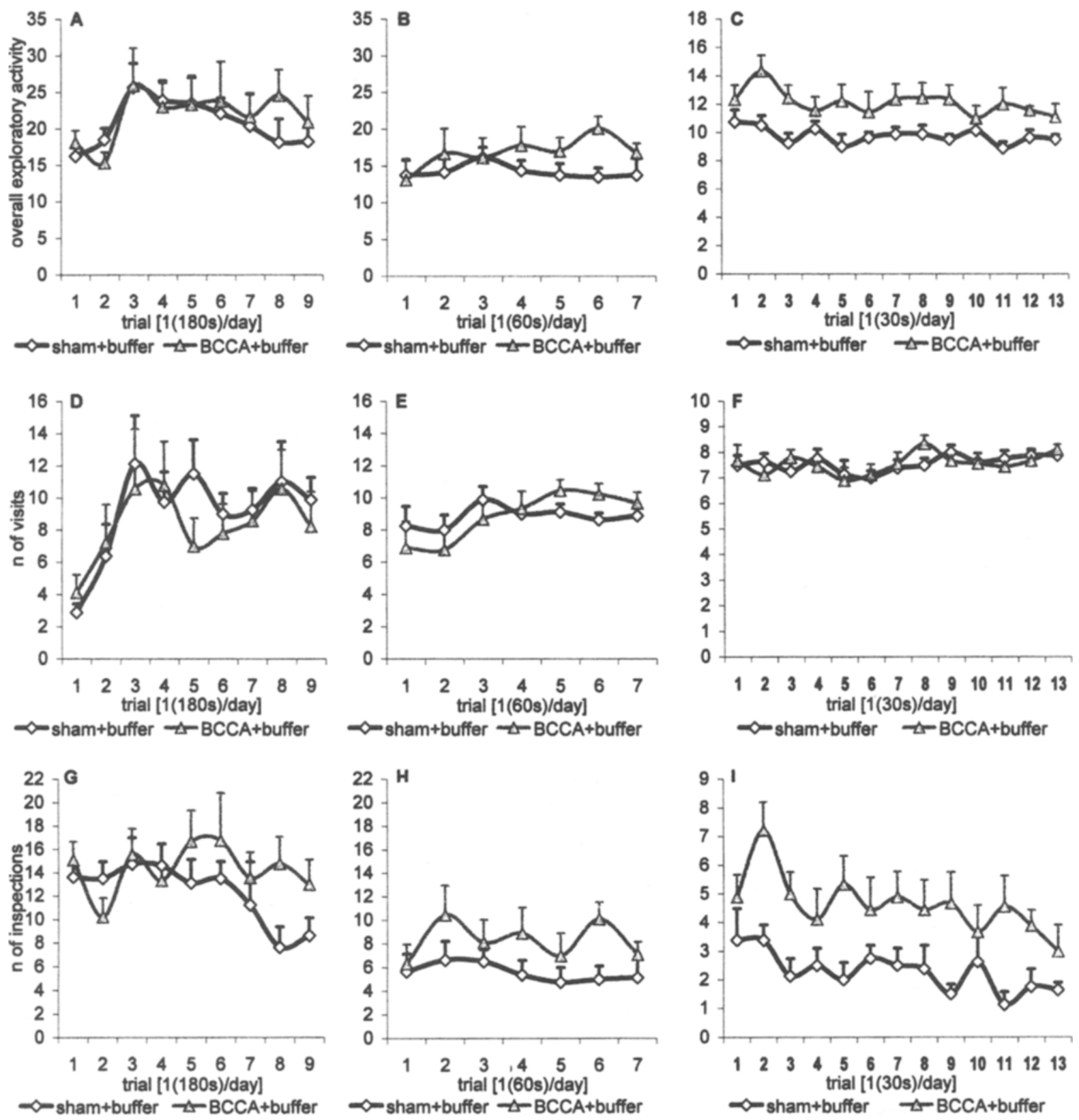

Figure 2. Curves illustrating overall exploratory activity in all three time periods (panels A-C) with increased exploratory activity shown by the BCCA-affected rats in panels B and $C$. The inspections (panels $G-I$ ) indicate short dipping of the head at the upper surface of the holes, in contrast to intensive searching for pellets deep inside the holes (visits; panels D-F). Values are shown as mean $\pm S E M$; sham + buffer, $n=8$ rats; BCCA + buffer, $n=9$ rats.

ber of pellets could be found but that they were not successful enough. Increasing the number of inspections, therefore, increased their chances of finding pellets. After the time was shortened to $60 \mathrm{sec}$ and, ultimately, to $30 \mathrm{sec}$, they were still able to find the pellets, but an increased effort was required for them to do so. Although their time to find the pellets was the same as that for the sham-operated animals (Figure 1C), BCCA rats inspected more holes (Figure 2I). They must, therefore, have increased their running speed, suggesting a switch in their search strategy. The increase in speed diminished their attention to the task and to the pattern and again resulted in a higher rate of inspections of unbaited holes. Animals with a high rate of unrewarding inspections were not concentrated on the pattern, which can be detected by a decreased serial collection of pellets. The rats appeared hectic.

BCCA animals seem to be more sensitive to the reduction in the time available for the task, since the increased values for inspections were significant only dur- 

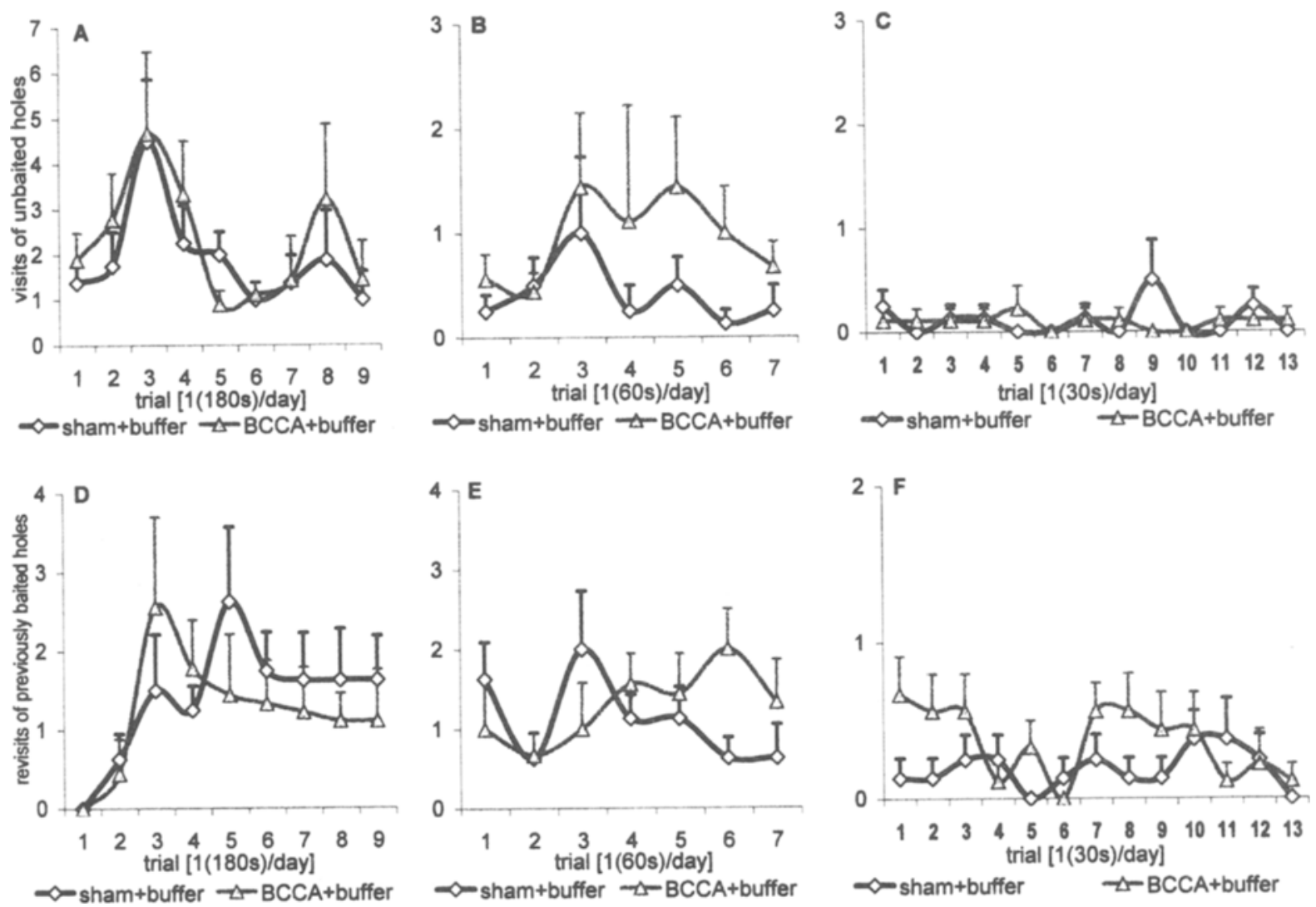

Figure 3. Curves that illustrate that both the control and the BCCA animals learned to avoid visits to unbaited holes (panels A-C). In panel B, a transient increase in visits to unbaited holes appears once again after reduction of the time available to $60 \mathrm{sec}$ per trial. In panel $\mathrm{C}$, both collections of rats avoided visits to unbaited holes, but the revisits to previously baited holes (panel F) was not completely avoided by the BCCA-affected rats, indicating that working memory errors were not avoidable. Values are shown as mean $\pm S E M$; sham + buffer, $n=8$ rats; $B C C A+$ buffer, $n=8$ rats.

ing the test periods with a reduced available time. Since, during the first trial sessions with $180 \mathrm{sec} /$ trial and $60 \mathrm{sec} /$ trial, sham-operated animals completed the task faster and, therefore, often ended the trial before the allotted time had elapsed, it might be argued that, for them, the defined time limit may have been irrelevant. In that case, only the BCCA animals would have been stressed by the time reduction and would, therefore, have displayed different behavior. However, the curves in Figures 1A-1C show that the means of both groups before the shortening of the time still lie above those for the next time limit allowed. After shortening the time (Figures $1 \mathrm{~B}$ and $1 \mathrm{C}$ ), the means of both groups varied around the time point of the allotted time or a little below it during the initial three trials (and the sham-operated animals were not always quicker), while the mean number of pellets eaten was still less than eight. This indicates that both BCCA and sham-operated animals could not successfully use up the whole of the available time and that both groups, therefore, became aware that time was limited.

The ability of individual rats to switch from Pattern $A$ to the new Pattern B, presented immediately after they had learned Pattern A, is shown in Figure 5. Rat 1 (Figure 5A) seems to be set on the previously learned Pattern A (Fig- ure $5 \mathrm{C}$, black quadrants): with the exception of Hole 3 , and Holes 19 and 20 on Day 3, and Hole 17 on Days 2 and 5, only holes belonging to Pattern A were inspected (Figure 5A, point in quadrant) or visited (cross in quadrant) up to the 5th experimental day. By using this unsuccessful search behavior, only two pellets were found (black filled quadrants) in Hole 1 (which belonged to both patterns) and Hole 3 , and seemingly, as a consequence of the negative feedback, the animal slackened searching. The negative effect seems to have induced a kind of learned helplessness.

Rat 2 (Figure 5B), on the other hand, very quickly abandoned the unsuccessful search. Exactly at the point at which Rat 1 had given up intensive searching, Rat 2 found six of the eight pellets (black filled quadrants), and furthermore, no more visits or inspections of any unbaited holes were recorded. The rat was completely successful in collecting and eating the pellets presented according to Pattern B.

Figure 5C shows the positions of the holes baited according to Pattern A (black) and Pattern B (gray).

A comparison of the slopes of the learning curves for pellets eaten for Pattern B (Figure 6, ascending), and the forgetting-curve for Pattern A, expressed as number of 

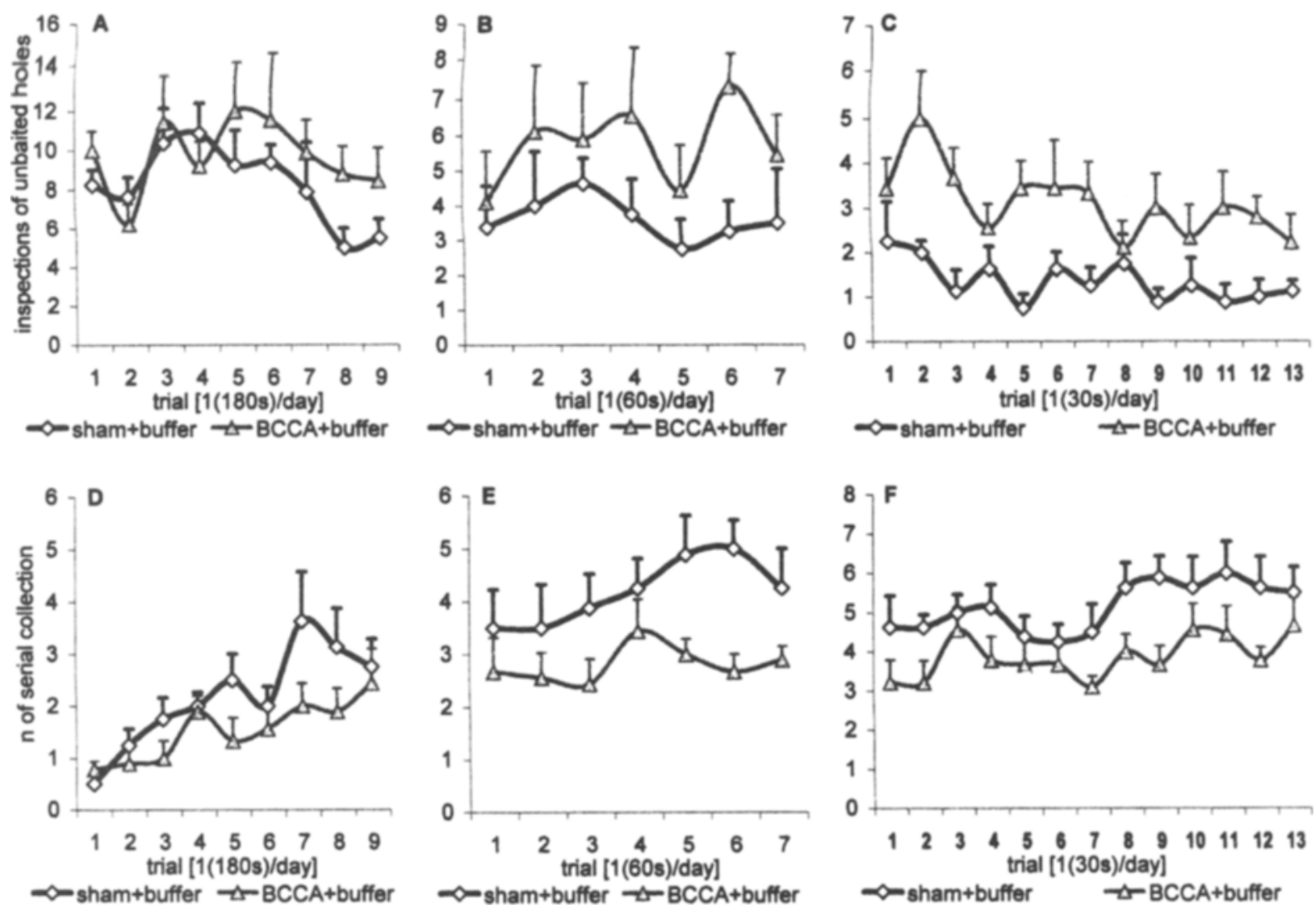

Figure 4. The curves in panels $A-C$ show the inspections of unbaited holes. BCCA-affected rats inspected more unbaited holes than did the controls, indicating that reference memory errors too could not be avoided. Panels $D-F$ demonstrate the capabilities of tested rats to find and eat the pellets in the given Pattern $A$ in a serial order without inspections or visits to holes not part of the pattern. The control rats learned to avoid mistakes better than did the BCCA-affected rats and appeared to be able to concentrate better on the learned pattern than did the BCCA-affected rats. Values are shown as mean $\pm S E M$; sham + buffer, $n=8$ rats; BCCA + buffer, $n=9$ rats.

inspected holes of the former pattern (Figure 6, descending), demonstrates a clear-cut distinction: BCCAtreated rats seem to have found it harder to relinquish their attachment to the former Pattern A and to switch to the new Pattern $B$, with which they found fewer pellets during the last three trials. On the first day with Pattern B presented, both BCCA-treated and sham-operated animals still inspected the same number of holes that were baited according to the previous Pattern A (sham, $4.63 \pm$ 0.38 ; BCCA, $4.67 \pm 0.82$ ). However, already on the 2 nd and 3rd day sham-operated animals reduced their inspections of Pattern A holes to a mean of $\sim 3$, a value that BCCA-treated animals arrived at only on the 6th day. Whereas an ANOVA revealed a significant difference for the forgetting curve of BCCA-treated rats, as compared with their sham-operated controls [Figure 6, descending; group effect, $F(1,15)=7.37, p=.0037$; trial effect, $F(10,150)=12.72, p=.0001$; no group $\times$ trial interaction], the learning curves for $\mathrm{B}$ showed no difference. However, only all the sham-operated rats succeeded in finding all of the pellets on the last experimental day. When the last three trials were separately calculated,
BCCA rats found significantly fewer pellets [group effect, Trials 9-11: $F(1,15)=6.70, p=.0129$ ].

Of the other parameters obtained (see Table 1), only a few showed significant differences. The overall exploratory activity was increased [Figure $7 \mathrm{~A}$; group effect, $F(1,15)=15.84, p=.0001$; no trial effect; no group $\times$ trial interaction; not shown], however, this was only obvious during the first 7 experimental days and resulted from an increased number of inspections [Figure 7B; group effect, $F(1,15)=20.05, p=.0001$; trial effect, $F(10,150)=7.39, p=.0001$; no group $\times$ trial interaction]. Figure $7 \mathrm{C}$ shows that inspections were devoted mostly to unbaited holes [group differences, $F(1,15)$ $=17.46, p=.0001$; trial effect, $F(10,150)=9.81, p=$ .0001 ; no group $\times$ trial interaction], and although ANOVA revealed a significant group difference for reinspections of previously baited holes [not shown; group effect, $F(1,15)=7.54, p=.0067$; no trial effect; no group $\times$ trial interaction], there was only an irrelevant increase observed among the BCCA rats (values around $n=1$ or less; not shown). The curve of visits to baited holes (not shown) showed decreased values for BCCA-treated rats, 
holes
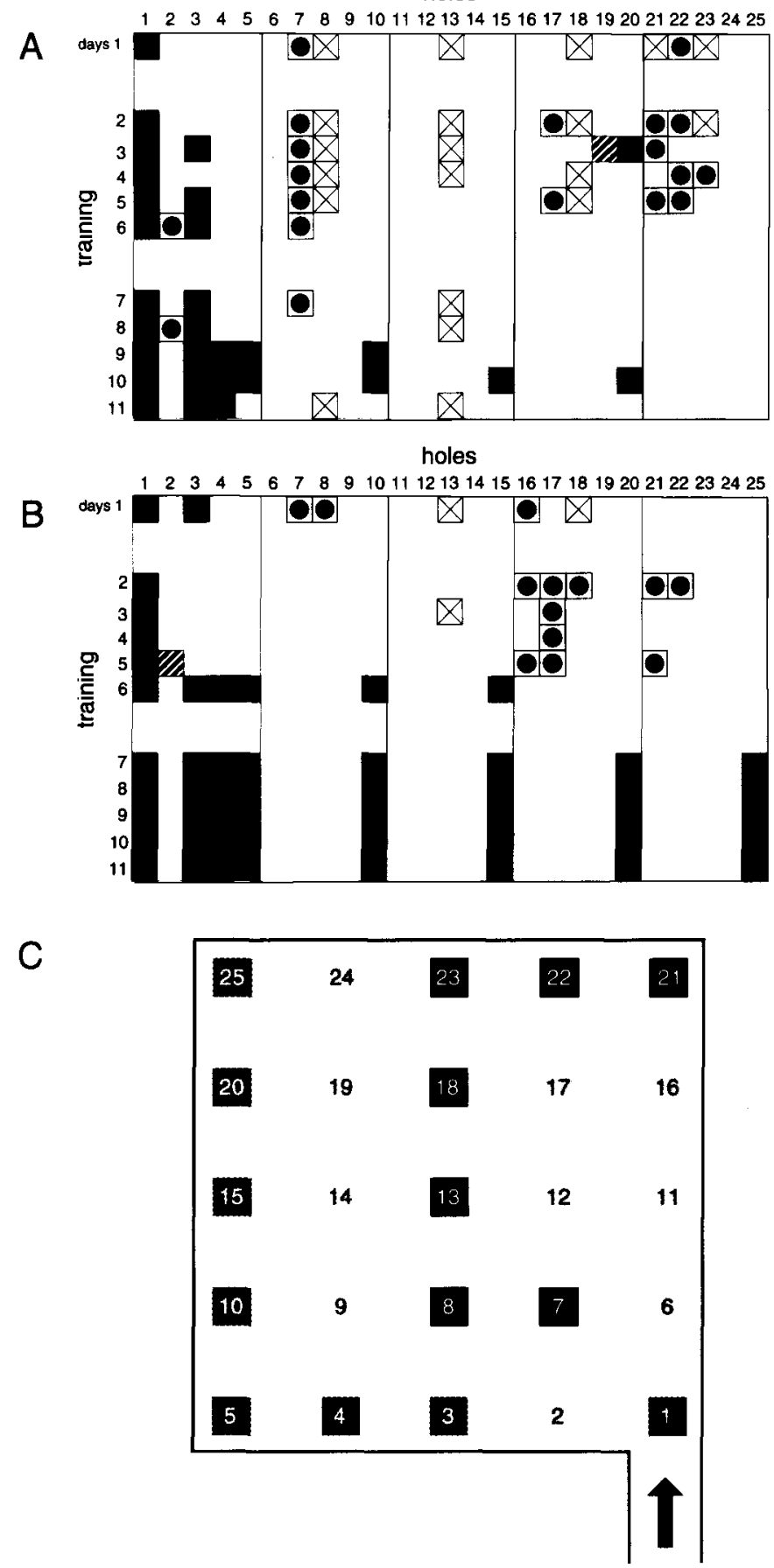

Figure 5. Detailed charts of the performance of 2 very different rats that were made to learn the new Pattern B immediately after initially learning Pattern $A$. C is a diagram of the hole board, in which Pattern A consists of Holes 1, 7, 8, 13, 18, 23, 22, and 21 and Pattern $B$ of Holes $1,3,4,5,10,15,20$, and 25 . Key to diagrams $A$ and $B$ : visits to holes previously baited according to the previous Pattern $A$ are indicated by crosses in quadrants; points in quadrants indicate inspections; hatched quadrants indicate visits to holes belonging to neither Pattern A nor B. The rat in diagram A, during training from Day 1 to Day 11 (vertical axis), by chance found a pellet in Hole 3 on Day 3 and on Days 5-11 (black fields), one pellet in Hole 20 on Day 3, one pellet in Hole 4 on Days 9-11, one pellet in Hole 5 and in Hole 10 on Days 9 and 10, and one pellet in Hole 15 and in Hole 20 on Day 10 (see horizontal axis). It does not appear that a learning process has taken place. The switching facility of the rat shown in diagram $B$ in relinquishing Pattern $A$ and concentrating on Pattern $B$ is, however, optimal. From Day 6 to Day 11 , the rat exactly followed Pattern B. The Hole 1 at the entrance is common to both Pattern A and Pattern B. 


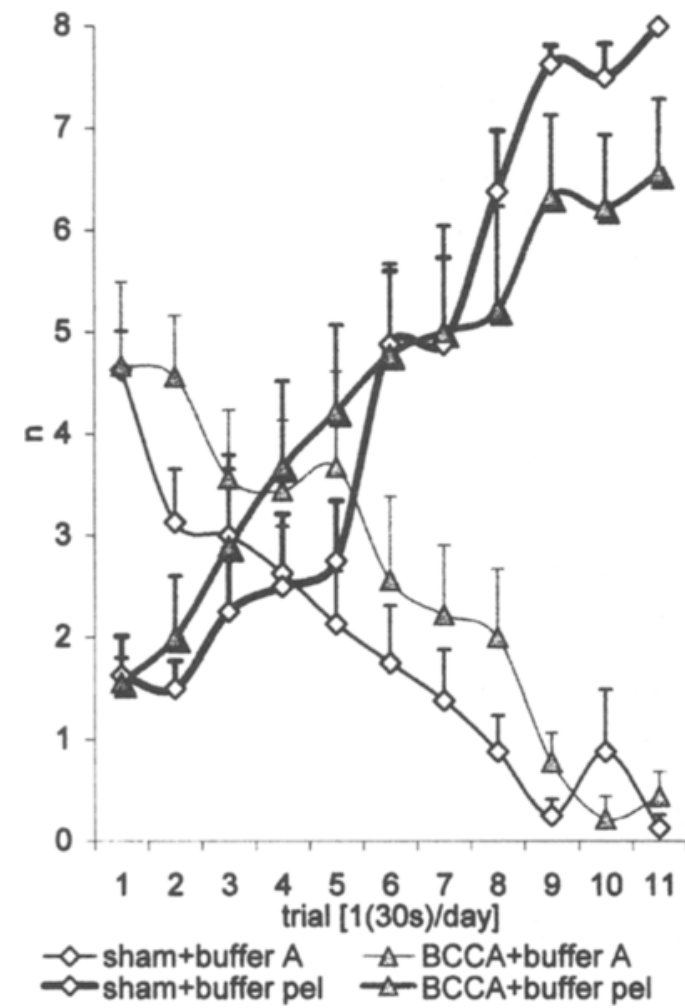

Figure 6. The forgetting curve of the previous Pattern A, together with the corresponding learning curve for the new Pattern B. The number of inspected holes of the former Pattern $A$ (descending curve) is shown in comparison with the number of pellets eaten (ascending curve) during the time course of 11 trials, 1 trial per day. BCCA-affected rats "forget" Pattern A later, as is shown by the comparison of the values between Days 5 and 6 , in contrast to the controls. The controls "forget" Pattern A 3 days earlier. There was no difference in the learning curves of the two groups, except for the values on Days 9-11. Values are shown as mean $\pm S E M$; sham + buffer, $n=8$ rats; $B C C A+$ buffer, $n=9$ rats.

compared with controls, during the last three trials [group effect, Trials 9-11: $F(1,15)=7.89, p=.0073$ ], suggesting that BCCA rats-like the animal shown in Figure $5 \mathrm{~A}-$ did not proceed far enough in following the pattern up till the last hole, No. 25. Reference memory errors, total [group effect, $F(1,15)=5.05, p=.0260$; trial effect, $F(10,150)=28.68, p=.0001$; no group $\times$ trial interaction; not shown] and inspections of unbaited holes in relation to overall exploratory activity [group effect, $F(1,15)=6.63, p=.0109$; trial effect, $F(10,150)=$ $17.17, p=.0001$; no group $\times$ trial effect; not shown] showed significant differences, suggesting that the unrewarding inspections of unbaited holes were not only due to an increase in overall exploratory activity, but also to failure of the reference memory, insofar as the rats could not "forget" a previously learned pattern quickly enough.

Additional treatment with iron in either brain structure, the striatum or the substantia nigra, had no significant effect on the overall learning capability of the rats: At the end of the 30-sec training session, all of the animals were completely successful in finding all of the pellets during the allotted time (not shown). However, as can be seen from Figure 8, striking differences were observed during the 180-sec acquisition period: BCCA-treated animals with additional intranigral iron (bcfeSN) immediately started increasing their visits already on the 2nd day, whereas intrastriatally iron-treated BCCA rats (bcfestria) did not start to increase their visits until the 6th experimental day [Figure 8A; group effect, $F(1,22)=$ $12.82, p=.0004$; trial effect, $F(8,176)=9.47, p=$ .0001 ; group $\times$ trial interaction, $F(8,176)=15.32, p=$ $.0001]$. BcfeSN rats showed the highest increase in their visits to unbaited holes (Figure 8B) on the 3rd day, but also an immediate, remarkable decrease on Days 4-5, whereas intrastriatally iron-treated BCCA rats (bcfestria) showed a continuously increasing number of visits to unbaited holes starting from Day 5 [no group effect; trial effect, $F(8,176)=2.6, p=.0102$; group $\times$ trial interaction, $F(8,176)=15.99, p=.0001]$. Whereas the percentage of reference memory errors decreased continuously for the bcfeSN rats, the curve for bcfestria rats showed a plateau at values of $\sim 50 \%$ over the whole 9-day period [Figure 8C; group effect, $F(1,22)=21.00, p=$ .0001 ; trial effect, $F(8,176)=4.26, p=.0001$; group $\times$ trial interaction, $F(8,176)=4.79, p=.0001]$. In Figure $8 \mathrm{D}$, the slopes of the curves for the number of pellets eaten show a steep increase for the bcfeSN rats, starting at Day 2, but the increase was retarded for the bcfestria rats [group effect, $F(1,22)=111.44, p=.0001$; trial effect, $F(8,197)=37.27, p=.0001$; group $\times$ trial interaction, $F(8,176)=7.13, p=.0001]$.

The time-to-learn curve in Figure 9, as an example, demonstrates the success of the bcfeSN group in the form of a typical sigmoidal curve. During the learning process, the time point at which the rats found half of the distributed pellets varied from one animal to another. If one employs a nonlinear transformation of all the individual time-to-learn curves to a central point at which all of the animals have found half of the pellets (time-tolearn fit), the experimenter is presented with a bundle set of very similar sigmoidal curves for any one experimental group (see Figure 9). The track of these curves reflects the learning success of the rats. Normally (and this was found for all the experimental groups mentioned here), the steepness of the curves reflect the all-or-nothing nature of the learning process. The time point for finding half the distributed pellets did, however, vary for the different groups investigated.

The results of the BCCA groups with additional injections of iron show a different behavior pattern for both bcfeSN and bcfestria animals during the initial learning phase, during which bcfeSN rats learned very quickly to abandon the wrong searching behavior of visiting unbaited holes, which is also apparent from the curve for reference memory errors. As the decrease in reference memory errors is accompanied by a steep increase in the number of pellets eaten, with a plateau from 

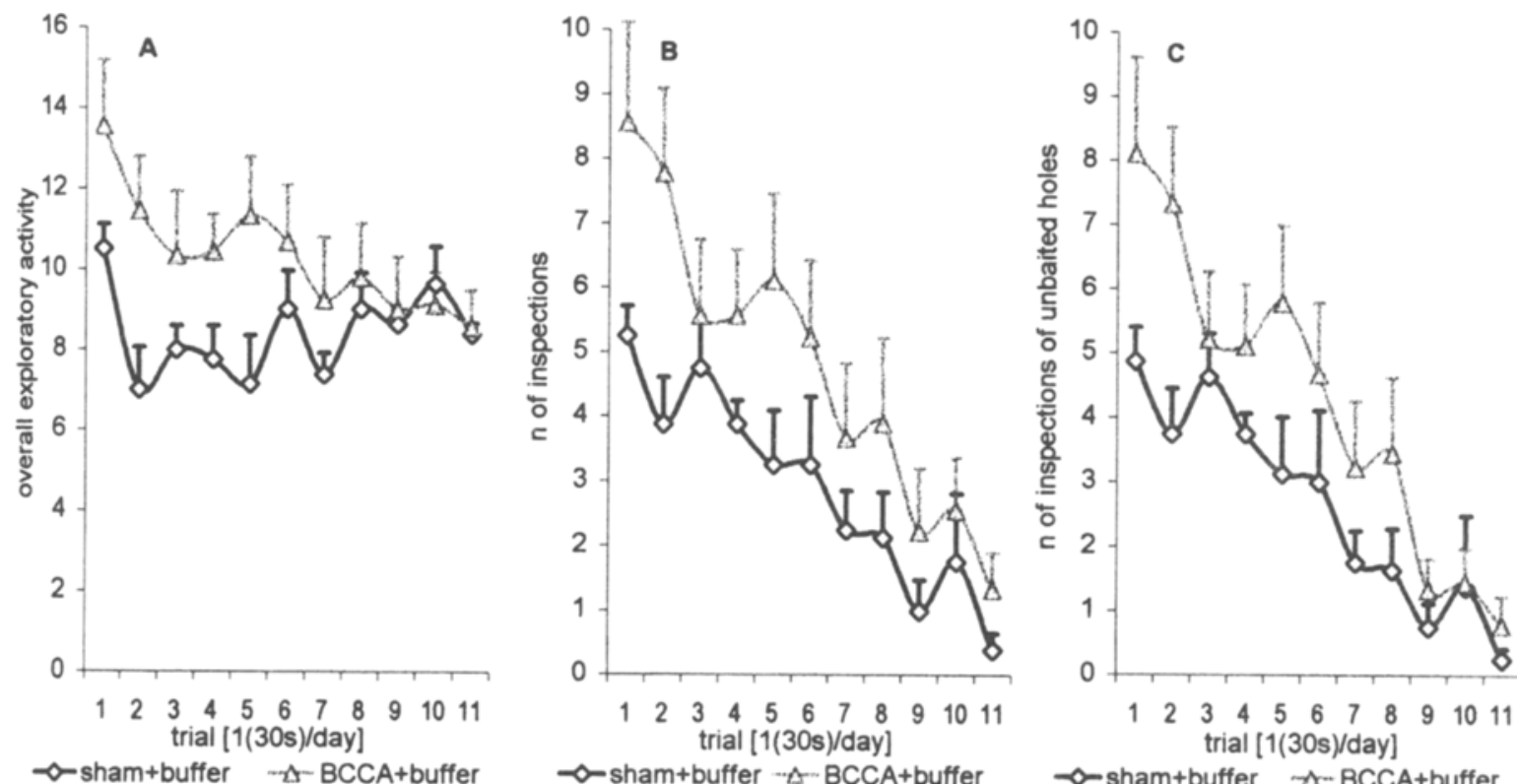

Figure 7. The curves of the higher overall exploratory activity of BCCA-affected rats, as compared with controls (panel A), during the learning period of Pattern B (afier Pattern $A$ had been learned) show that this activity was spent in inspecting holes, rather than in visiting holes, which would have offered the opportunity of actually finding pellets there (panel $B$ ). Inspections were devoted almost exclusively to unbaited holes (panel $C$ ). Values are shown as mean $\pm S E M$; sham + buffer $n=8$ rats; BCCA + buffer $n=9$ rats.

the 4th until the final experimental day, it can be assumed that the rats' success was achieved by virtue of a cognitive process (see also Figure 9) and economical collecting (the black horizontal line in panels $\mathrm{A}$ and $\mathrm{D}$ of Figure 8 marks the economical collection period), using their reference memory with the help of intraboard and distal spatial cues, as well as egocentric coordinates (a distinction between the cues used cannot be made by using only the parameters listed in Table 1).

Bcfestria rats, however, were not able to reduce their visits to unbaited holes during the course of the trials. The lower overall exploratory activity observed for the bcfestria group, as compared with bcfeSN [group effect, $F(1,22)=40.98, p=.0001$; trial effect, $F(8,176)=4.54$, $p=.0001$; group $\times$ trial interaction, $F(8,176)=7.82$, $p=.0001$; not shown] cannot by itself account for their poorer performance of the task. The diminished exploratory activity may account for the small number of visits (Figure 8A), preventing the animals from finding pellets, and may have served to retard their success (Figure 8D). However, the curve of reference memory errors (Figure $8 \mathrm{C}$ ) clearly shows that the late increase in exploratory activity cannot alone be used for rewarding visits. Although the time-to-learn curve (see Figure 9) revealed a comparable capability for recognition of the task (not shown) and their success at finding the hidden pellets reached that of the bcfeSN rats (Figure 8D), bcfestria animals searched more uneconomically, suggesting that they have a poorer reference memory.

\section{DISCUSSION}

The data provided by COGITAT permit the identification of cognition and recognition, of learning and memory capabilities, and of orientation capabilities within the laboratory environment, as well as of search strategies using intraboard and distal cues and egocentric strategies. Using COGITAT, detailed analyses of the individual parameters involved in the performance of the task make it clear that the animals, during their exploration in several daily sessions, learned the pattern, using their individual behavior patterns. The present experiments were able to detect changes in behavioral measures related to exploration, and memory, and the shifting aptitude as a response to specific experimental manipulations.

\section{Methodological Aspects}

Some of the experimental data could also be obtained by other well-established methods. The question may arise whether the hole board system reveals behavioral information that could not be displayed by other systems, such as the water maze or the radial arm maze. There is a fundamental distinction between the water maze, on the one hand, and the radial arm maze and the hole board, on the other. The stress in the water maze forces the animals to use the best strategy that lets them escape from the water as quickly as possible (see also Brucato et al., 1996). Both the radial arm maze and the hole board, however, offer the animals the opportunity of exploring 

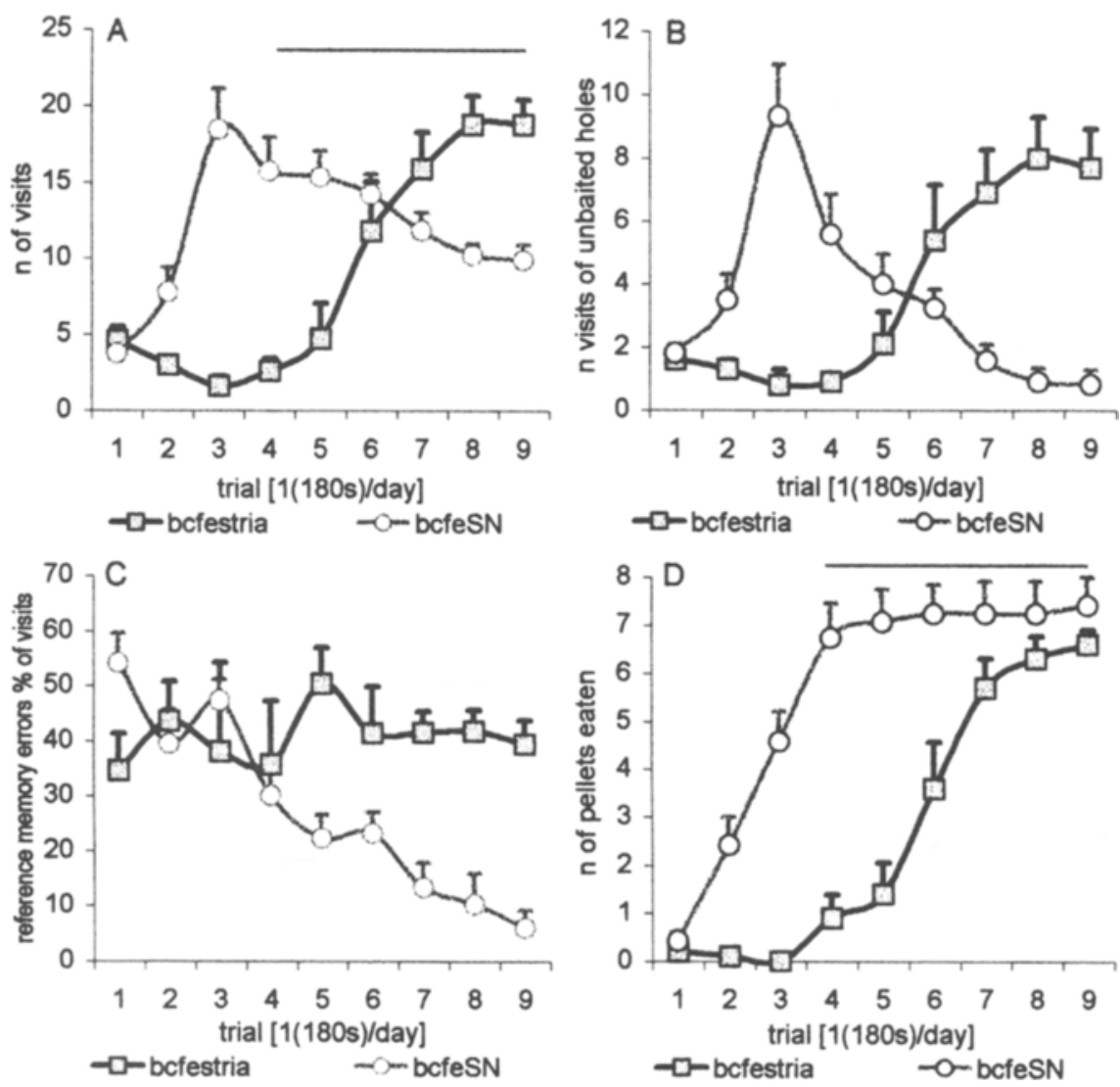

Figure 8. Acquisition of the food search task over 9 daily trials with Pattern A presented for 180 sec to animals that had received additional $\mathrm{FeCl}_{3}$ intrastriatally (bcfestria) or intranigrally (bcfeSN) 1 week after BCCA. Panel A: number of visits; panel B: number of visits to unbaited holes; panel $C$ : number of visits to unbaited holes as a percentage of the total number of visits, which is equivalent to reference memory errors; panel D: number of pellets eaten. Values are shown as mean $\pm S E M$; bcfestria, $n=12$ rats; bcfeSN, $n=12$ rats. Panel A: BcfeSN rats showed a high exploratory activity (visits) on Day 3 , whereas befestria rats developed increased exploratory activity later. The peak of the befeSN rats in panel $B$ correlated with visits (panel A). Maximal success in finding and eating pellets in panel $D$ had already been achieved 1 day later, which was related to a drastic decrease in visits to unbaited holes (panel B) and with a decrease in the percentage of reference memory errors of visits (panel C); this means that the rats learned to avoid visiting unbaited holes. By contrast, the befestria rats, over a 9-day period, always made the same number of errors, expressed as a percentage of visits (panel $\mathrm{C}$ ), so these rats had to work harder for the same success.

the new environment and, after habituation, of finding food. On the hole board, in particular, rats (and mice) display exploratory behavior (Fiore et al., 1998; Thullier, Lalonde, Mahler, Joyal, \& Lestienne, 1996), as well as food-searching behavior (Oades, 1981), without being forced to do so. (This is also suggested by our earlier observation that, when the rats knew that they were going to be given their entire daily ration of food after the trial anyway, they did not search for the pellets in the hole board sufficiently eagerly.) Therefore, the water maze, and the radial maze and/or the hole board, are measuring different cognitive procedures and reveal different expressions of behavioral items and impairments (Dellu, Mayo, Vallee, Le Moal, \& Simon, 1997; Nelson, Lebessi, Sowinski, \& Hodges, 1997; Oades, 1981; Ohta, Nishikawa,
Kimura, Anayama, \& Miyamoto, 1997; Schwegler et al., 1996). Furthermore, reference memory errors and/or working memory errors measured in the radial maze or with the hole board are not validly comparable (see van Luijtelaar, van der Staay, \& Kerbusch, 1989).

The COGITAT system records multiple behavioral items simultaneously, which are displayed immediately after the end of the experiment. Furthermore, the timeto-learn fit provides at a glance information about the capability of the tested groups to recognize the task. Up to now, with the use of the experimental groups presented here, no changes in the steepness of the curves have been recorded, and the steepness of the slope warrants the conclusion of an all-or-nothing effect. It would, however, be very interesting to see whether this effect would be af- 


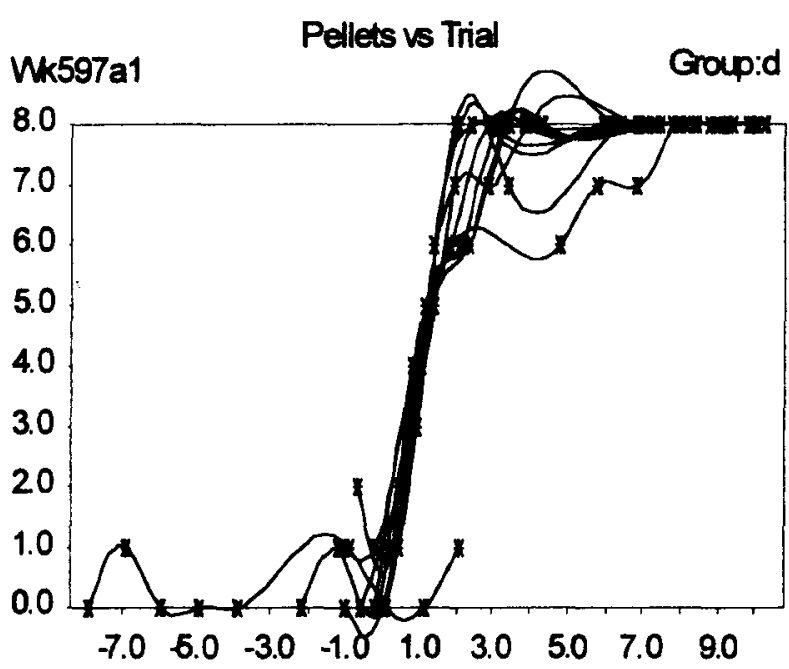

Figure 9. The time-to-learn curve shows the success of all the animals of the bcfeSN group. The steep slope of these curves indicates a learning capability within the normal range (see the text). Abscissa, -7 to 0 : the delay from the start of the experiment until half the pellets have been found; 0 is the time point at which half the pellets have been found; ordinate: the number of pellets eaten.

fected by distinct neurophysiological, neuropharmacological, or gene-technological interventions.

The use of different and distinct test procedures demonstrated that each of them showed different brain structures and/or transmitter systems to be involved that were not uncovered by the other (Brucato et al., 1996; Oades, 1981; Schwegler et al., 1996). From our own experiments (Heim, Zhang, et al., 2000), it could be shown that no differences between sham-operated and BCCA-treated rats were detectable when various different parameters were measured 3 months post surgery in a water maze (Morris, Garrud, Rawlins, \& O'Keefe, 1982), suggesting that there were no reference memory deficits. COGITAT, however, showed up differences between the groups, although these differences were very subtle ones. Furthermore, when tested in an open field (Heim et al., 1999), BCCAtreated rats showed somewhat decreased locomotor and exploratory activity during the first $4 \mathrm{~min}$ of observation, as compared with controls, whereas on the hole board, there was no difference between the groups, and, after shortening the time available for the task, an increase in exploratory activity could even be recorded. These examples point up the necessity of recording multiple parameters simultaneously for each distinct test procedure.

\section{Scientific Aspects}

Progressive neurodegenerative disease, such as the consequences of cerebral oligemia, stroke, or oxidative stress after hydroxyl free radical production, involves more than one system, and the interplay of pathophysiologically functioning systems may shift at different stages of the disease, being at times scarcely detectable. For these rea- sons, when testing animal models of neurodegenerative diseases, an analytical system is required that is able to measure as many behavioral parameters as possible simultaneously, so that the experimenter can not only detect that the animals do demonstrate deficits, but can also obtain detailed insights into the nature of these processes and into the structures and systems involved in theșe deficits and in the different distinct behavior patterns used by the animals to compensate for them.

During the experimental procedure demonstrated here, the consequences of two different types of cerebral interventions were investigated, in order to record the whole behavioral profile of affected rats after an oligemic episode (BCCA) or after being additionally injected with iron, either into the substantia nigra or into the ventrolateral striatum-a constellation that plays a role in aging brain structures and has been suggested to be involved in the aging process (Lauffer, 1992; Martin, Ye, \& Allen, 1998; Ostrow \& Miller, 1993). Both interventions may initiate processes that lead to the development of neurodegenerative disorders (Arendash et al., 1993; Pasquier, Leys, \& Scheltens, 1998; M. A. Smith, Harris, Sayre, \& Perry, 1997).

BCCA stimulates the release of dopamine, as was evidenced for the ventrolateral striatum (Heim, Zhang, et al., 2000; Läer, Block, Huether, Heim, \& Sontag, 1993), which leads to the generation of highly toxic hydroxyl radicals (Heim, Zhang, et al., 2000). It is also known that BCCA leads to increased lipid peroxidation in cortical, striatal, and hippocampal tissues, which, in the striatum and the hippocampus, can be potentiated by iron injection into the ventrolateral striatum (Heim et al., 1995; Melzacka et al., 1995; Melzacka et al., 1994). Furthermore, the BCCA procedure leaves decreased dopamine DIRmRNA, as determined 19 months after the BCCA procedure, evidenced by in situ hybridization in slices of the dorsolateral striatum (Heim et al., 1999). It has been suggested that striatal dopamine D1-like receptors play a critical role in incentive learning (Beninger \& Miller, 1998).

BCCA, in combination with additional intrastriatal iron, may also affect structures that are involved in circuits (see Depue \& Collins, 1999), including the shell of the nucleus accumbens (NASshell), the ventrolateral subterritory of the ventral pallidum, and the ventrotegmental area, with their dopamine ascending projections (analyses of BCCAaffected tissues are in preparation). Because the interdependent region--that is, the NASshell, the ventrolateral subterritory of the ventral pallidum, and the ventrotegmental area with their dopamine ascending projectionselicit the initiation of locomotor activity (Depue \& Collins, 1999; Kalivas, Churchill, \& Klitenick, 1993; Klitenick, Deutch, Churchill, \& Kalivas, 1992; Koob, Robledo, Markou, \& Caine, 1993), a damaging effect in these areas would explain why such additionally iron-injected BCCA rats exhibited a diminished initial locomotor activity. The target point for the injected iron within the ventrolateral striatum is not far from the NASshell, and is within 
the reciprocal pathways between the NASshell and the hippocampal and extended amygdala (see also Groenewegen et al., 1991). Moreover, the ventrolateral striatum is functionally strongly connected with the nucleus accumbens (Koshikawa, Yoshida, Kitamura, Saigusa, \& Cools, 1996). The oxidative stress induced by additional iron within these areas may potentiate the effects of oligemia by destroying the reciprocal connections, afferents, and efferents of the NASshell with the extended amygdala, basal lateral complex, and hippocampus because of the iron deposit and the track of the injection needle within the ventrolateral striatum. The consequence would be less initiation of locomotion by the nucleus accumbens (see Kalivas et al., 1993), because of diminished motivational information arriving from the amygdala and the hippocampus (Mogenson, Grudzynski, Wu, Yang, \& Yim, 1993).

In comparison with the BCCA group with additional intrastriatal iron injection, the rats that received the iron injection into the substantia nigra (bcfeSN) appear to have neither cognitive, nor motor, nor motivational deficiencies (Figures 8A-8D). However, if one looks at the results of the multiple factorial analyses of their behavior obtained by COGITAT (not shown in detail here; results in preparation) regarding their overall exploratory activity and number of visits, it turns out that the bcfeSN rats had increased and the bcfestria rats had decreased exploratory activities, as compared with their corresponding controls.

Also, when compared with the bcfeSN rats, the bcfestria rats exhibited lower overall exploratory activity (not shown). A comparison of the curves for both groups in Figure 8 shows that the nigra animals appear to have a better reference memory (Figure $8 \mathrm{C}$ ) than do the striatum animals. It seems that the positive information - that is, the early success in finding food because of the increased number of visits (Figure 8A)-produced a positive bias. The absence of intensive searching (i.e., visits) on the part of the striatally affected animals, on the other hand, seems to be responsible for a smaller benefit from the information.

Our conclusion, drawn from Figure 8, is that the increased number of visits, combined with a steep increase in the number of unbaited holes, provides a cognitive profit and that the immediate subsequent drastic fall represents a cognitive process and an excellent learning effect, and this view gains support from the most recent reports by Manahan-Vaughan and Braunewell (1999). These authors used a mini-hole board and were able to show that novelty acquisition (which was combined with a significant decrease in exploratory behavior after reexposure to the hole board) facilitated long-term depression (LTD) in the hippocampal CAl region of freely moving rats. Exploratory behavior may correspond to a form of information acquisition (Eichenbaum, 1996; O'Keefe \& Nadel, 1978), whereas evidence of habituation is believed to represent proof that learning has taken place (File \& Wardill, 1975; Platel \& Porsolt, 1982). LTD may be regarded as a mechanism for memory (Bear, 1999).
A comparison of these two animal models leads to the conclusion that both of them exhibited signs characterizing neurodegenerative processes detectable by a multifactorial analysis, but, for the differentiation of distinct and specific behavior items, the experimenter is obliged to record behavior items simultaneously and, also, to observe the further developments of behavior items over a long period of time, as well as their differential predominance at distinct time periods.

In summary, the COGITAT system provides information concerning specific deficiencies. The automatic and computerized simultaneous analysis of a variety of behavioral parameters, together with their statistical and graphical presentation, offers an objective characterization of cognitive, memory, and learning capabilities, as well as of behavior patterns involved in incentive learning, and of the capabilities of rats to switch between different locations where food is presented, using different behavior patterns. The system measures in a single run multiple behavioral items that can otherwise only be collected during different and consecutive test procedures.

\section{REFERENCES}

Arendash, G. W., Olanow, C. W., \& Sengstock, G. J. (1993). Intranigral iron infusion in rats: A progressive model for excess nigral levels in Parkinson's disease? In P. Riederer \& W. Wesemann (Eds.), Iron in central nervous system disorders (pp. 86-101). Vienna: SpringerVerlag.

BEAR, M. F. (1999). Homosynaptic long-term depression: A mechanism for memory? Proceedings of the National Academy of Sciences, 96, 9457-9458.

Beninger, R. J., \& Miller, R. (1998). Dopamine Dl-like receptors and reward-related incentive learning. Neuroscience \& Biobehavioural Reviews, 22, 335-345.

Block, F., Sieklucka, M., Schmidt-Kastner, R., Heim, C., \& SonTAG, K.-H. (1993). Metabolic changes during and after transient clamping of carotid arteries in normotensive rats. Brain Research Bulletin, 31, 91-96.

Block, F., Szabo, L., Jaspers, R. M. A., Heim, C., \& Sontag, K.-H. (1993). Levemopamil reduces spatial learning deficit following transient occlusion of common carotid arteries in normotensive rats. Acta Neurologica Scandinavica, 88, 35-40.

Brucato, F. H., Levin, E. D., Mott, D. D., Lewis, D. V., Wilson, W. A., \& SWARTZWELDER, H. S. (1996). Hippocampal long-term potentiation and spatial learning in the rat: Effects of $G A B A B$ receptor blockade. Neuroscience, 74, 331-339.

ConNor, J. R. (1992). Proteins of iron regulation in the brain in Alzheimer's disease. In R. B. Lauffer (Ed.), Iron and human disease (pp. 2-20). Boca Raton, FL: CRC Press.

Dellu, F., Mayo, W., Vallee, M., Le Moal, M., \& Simon, H. (1997). Facilitation of cognitive performance in aged rats by past experience depends on the type of in formation processing involved: A combined cross-sectional and longitudinal study. Neurobiology of Learning \& Memory, 67, 121-128.

Depue, R. A., \& Collins, P. F. (1999). Neurobiology of the structure of personality: Dopamine, facilitation of incentive motivation, and extraversion. Behavioural \& Brain Sciences, 22, 491-569.

Deroche, V., Marinelli, M., Maccari, S., Le Moal, M., Simon, H., \& PiAzZA, P. V. (1995). Stress-induced sensitization and glucocorticoids: I. Sensitization of dopamine-dependent locomotor effects of amphetamine and morphine depends on stress-induced Corticosterone secretion. Journal of Neuroscience, 15, 7181-7188.

Eichenbaum, H. (1996). Is the rodent hippocampus just for 'place'? Current Opinion on Neurobiology, 6, 187-195.

EiJKenboom, M., \& VAN DER STAay, F. J. (1998). Effects of brain le- 
sions on Morris water maze performance in rats: Inter- and intraexperiment variability [Abstract]. In L. P. J. J. Noldus (Ed.), Measuring Behavior '98: Proceedings of the 2nd International Conference on Methods and Techniques in Behavior Research (pp. 139-140). Wageningen: Noldus Information Technology.

FILE, S. E., \& WARDILL, A. G. (1975). Validity of head-dipping as a measure of exploration in a modified hole-board. Psychopharmacology, 44, 53-59.

Fiore, M., Alleva, E., Probert, L., Kollias, G., Angelucci, F., \& ALOE, L. (1998). Exploratory and displacement behaviour in transgenic mice expressing high levels of brain TNF-alpha. Physiology \& Behaviour, 63, 571-576.

Groenewegen, H., Berendse, H., Meredith, G., Haber, S., Voorn, P., WOLTERS, J., \& LOHMANN, A. (1991). Functional anatomy of the ventral, limbic system-innervated striatum. In P. Willner \& J. ScheelKrùger (Eds.), The mesolimbic dopamine system: Motivation to action (pp. 19-59). New York: Wiley.

Heim, C., Arzberger, T., Sontag, T., Xiao, A., Herbinger, K.-H., Weindl, A., \& Sontag, K.-H. (1999). Progressive degeneration of dopamine system functions after transient cerebral oligemia in rats. Brain Research, 851, 235-246.

Heim, C., Kolasiewicz, W., \& Sontag, K.-H. (2000). The effects of the 21 -aminosteroid $\mathrm{U}-74389 \mathrm{G}$ on spatial orientation in rats after a cerebral oligemic episode and iron-induced oxidative stress. Journal of Neural Transmission, 107, 95-104.

Heim, C., Melzacka, M., Kolasiewicz, W., Jaros, T., Sieklucka, M., Wesemann, W., \& Sontag, K.-H. ( 1995 ). Cerebral oligemic hypoxia and iron toxicity in the mesolimbic system of rats. Journal of Neural Transmission, 46(Suppl.), 165-173.

Heim C., \& SonTAg, K.-H. (1994). Reference memory is affected by transient bilateral clamping of the carotid arteries in rats (BCCA). Journal of Neural Transmission, 7, 47-59.

Heim, C., Zhang, J., Lan, J., Sieklucka, M., Kurz, T., Riederer, P., Gerlach, M., \& SonTaG, K.-H. (2000). Cerebral oligaemia episode triggers free radical formation, and late cognitive deficiencies. $E u$ ropean Journal of Neuroscience, 12, 1-11.

Kalivas, P., Churchill, L., \& Klitenick, M. (1993). The circuitry mediating the translation of motivational stimuli into adaptive motor responses. In P. Kalivas \& C. Barnes (Eds.), Limbic motor circuits and neuropsychiatry (pp. 237-287). Boca Raton, FL: CRC Press.

Klitenick, M., Deutch, A., Churchill, L., \& Kalivas, P. (1992). Topography and functional role of dopaminergic projections from the ventral mesencephalic tegmentum to the ventral pallidum. Neuroscience, 50, 371-382.

Kolasiewicz, W., Jaros, T., Heim, C., Melzacka, M., Sieklucka, M., Weiner, N., WesemanN, W., Riederer, P., \& Sontag, K.-H. (1995). Injection of a minuscule dose of $\mathrm{FeCl}_{3}$ within the ventrolateral striatum causes a chronic disturbance of the integrative function within the limbic part of the ventral striatum. Journal of Neural Transmission, 9, 15-29.

Koob, G., Robledo, P., Markou, A., \& Caine, S. B. (1993). The mesocorticolimbic circuit in drug dependence and reward: A role for the extended amygdala? In P. Kalivas \& C. Barnes (Eds.), Limbic motor circuits and neuropsychiatry (pp. 289-309). Boca Raton, FL: CRC Press.

Koshikawa, N., Yoshida, Y., Kitamura, M., Saigusa, T., \& Cools, A. R. (1996). Behavioural and biochemical stimulation in the nucleus accumbens differentially alters dopaminergic activities in rat ventrolateral striatum. In C. Ohye, M. Kimura, \& J. S. McKenzie (Eds.), Advances in behavioral biology: Vol. 47. The basal ganglia V (pp. 105111). New York: Plenum.

Läer, S., Block, F., Huether, G., Heim, C., \& Sontag, K.-H. (1993). Effect of transient reduction of cerebral blood flow in normotensive rats on striatal dopamine release. Journal of Neural Transmission, 92 , 203-211.

LAUFFER, R. B. (1992). Iron, aging, and human disease: Historical background and new hypotheses. In R. B. Lauffer (Ed.), Iron and human disease (pp. 2-20). Boca Raton, FL: CRC Press.
Manahan-Vaughan, D., \& Braunewell, K.-H. (1999). Novelty acquisition is associated with induction of hippocampal long-term depression. Proceedings of the National Academy of Sciences, 96, 8739-8744.

MARTIN W. R., YE, F. Q., \& ALLEN, P. S. (1998). Increasing striatal iron content associated with normal aging. Movement Disorders, 13, 281-286.

Melzacka, M., Heim, C., Kolasiewicz, W., Sieklucka, M., Jaros, T., Wesemann, W., \& Sontag, K.-H. (1995). Cerebral oligemic hypoxia during bilateral clamping of the carotid arteries prolongates ironinduced lipid peroxidation. Journal of Neural Transmission, 102, 31-32.

Melzacka, M., Weiner, N., Heim, C., Schmidt-Kastner, R., Sieklucka, M., Sontag, K.-H., \& WesemanN, W. (1994). Effect of a transient reduction of cerebral blood flow on membrane anisotropy and lipid peroxidation in different rat brain areas. Neurochemistry International, 25, 161-168.

Mogenson, G., Grudzynski, S., Wu, M., YANG, C., \& YiM, C. (1993) From motivation to action. In P. Kalinas \& C. Barnes (Eds.), Limbic circuits and neuropsychiatry (pp. 193-236). Boca Raton, FL: CRC Press.

Morris, R. G. M., Garrud, P., RaWlins, J. N. P., \& O'KeEFE, J. (1982). Place navigation impaired in rats with hippocampal lesions. Nature, 297, 681-683.

Nakano, S., Kogure, K., \& Fujikura, H. (1990). Ischemia-induced slowly progressive neuronal damage in the rat brain. Neuroscience, 38, $115-124$.

Nelson A., Lebessi A., Sowinski P., \& Hodges, H. (1997). Comparison of effects of global cerebral ischaemia on spatial learning in the standard and radial water maze: Relationship of hippocampal damage to performance. Behavioural Brain Research, 85, 93-115.

OADES, R. D. (1981). Impairments of search behaviour in rats after haloperidol treatment, hippocampal or neocortical damage suggest a mesocorticolimbic role in cognition. Biological Psychology, 12, 77-85.

OADES, R. D., \& ISAACSON, R. L. (1978). The development of food search behaviour by rats: The effects of hippocampal damage and haloperidol. Behavioural Biology, 24, 327-337.

Ohta, H., Nishikawa, H., Kimura, H., Anayama, H., \& Miyamoto, M. (1997). Chronic cerebral hypoperfusion by permanent internal carotid ligation produces learning impairment without brain damage in rats. Neuroscience, 79, 1039-1050.

O'KeEFE, J., \& NADEL, L. M. (1978). The hippocampus as a cognitive map. Oxford: Oxford University Press, Clarendon Press.

Ostrow P. T., \& Miller L. L. (1993). Pathology of small artery disease. Advances in Neurology, 62, 93-123.

Pasquier, F., Leys, D., \& Scheltens, P. (1998). The influence of coincidental vascular pathology on symptomatology and course of Alzheimer's disease. Journal of Neural Transmission, 54(Suppl.), 117-127.

Paxinos, G., \& WATSON, C. (1982). The rat brain in stereotaxic coordinates. New York: Academic Press.

Platel, A., \& Porsolt, R. D. (1982). Habituation of exploratory activity in mice: A screening test for memory enhancing drugs. Psychopharmacology, 78, 346-352.

Pothos, E. N., Creese, I., \& Hoebel, B. G. (1995). Restricted eating with weight loss selectively decreases extracellular dopamine in the nucleus accumbens and alters dopamine response to amphetamine, morphine, and food intake. Journal of Neuroscience, 15, 6640-6650.

Schwegler, H., Boldyreva, M., Linke, R., WU, J., Zilles, K., \& Crusio, W. E. (1996). Genetic variation in the morphology of the septohippocampal cholinergic and GABAergic systems in mice: II. Morphobehavioural correlations. Hippocampus, 6, 535-545.

Sengstock, G. J., Olanow, C. W., Dunn, A. J., \& Arendash, G. W. (1992). Iron induces degeneration of nigrostriatal neurons. Brain Research Bulletin, 28, 645-649.

Smith, M. L., Auer, R. B., \& SiesJö, B. K. (1984). The density and distribution of ischemic brain injury in the rat following 2-10 min of forebrain ischemia. Acta Neuropathologica, 64, 127-129.

Smith, M. A., Harris, P. L., Sayre, L. M., \& Perry, G. (1997). Iron accumulation in Alzheimer disease is a source of redox-generated free radicals. Proceedings of the National Academy of Sciences, 94, 9866-9868. 
Spruit, B. M., Buma, M. O. S., van Lochem, P. B. A., \& Rouseau, J. B. I. (1998). Automatic behavior recognition: What do we want to recognize and how do we measure it? [Abstract]. In L. P. J. J. Noldus (Ed.), Measuring Behavior '98: Proceedings of the 2nd International Conference on Methods and Techniques in Behavior Research (pp. 264-266). Wageningen: Noldus Information Technology.

Thullier, F., Lalonde, R., Mahler, P., Joyal, C. C., \& Lestienne, F. (1996). Dorsal striatal lesions in rats: 1 . Effects on exploration and motor coordination. Archives of Physiology \& Biochemistry, 104, 300-306.

van Luijtelaar, E. L., van der Staay, F. J., \& Kerbusch, J. M. (1989). Spatial memory in rats: A cross validation study. Quarterly Journal of Experimental Psychology, 41B, 287-306.

(Manuscript received December 18, 1998 revision accepted for publication October 3, 1999.) 